\title{
Second-harmonic generation as characterization tool for Ge/high-k dielectric interfaces
}

\author{
Maarten K. Vanbel ${ }^{\mathrm{a}}$, Annelies Delabie ${ }^{\mathrm{b}}$, Sonja Sioncke ${ }^{\mathrm{b}}$, Christoph Adelmann ${ }^{\mathrm{b}}$, Valeri V. \\ Afanas'ev $^{\mathrm{c}}$, Jean-Pierre Locquet ${ }^{\mathrm{d}}$, Sven Van Elshocht ${ }^{\mathrm{b}}$, Matty Caymax ${ }^{\mathrm{b}}$, Thierry Verbiest ${ }^{\mathrm{a}}$ \\ ${ }^{a}$ Molecular and Nanomaterials, Dept. of Chemistry, KU Leuven, Celestijnenlaan 200D, 3001 \\ Leuven, Belgium \\ ${ }^{\mathrm{b}}$ Imec, Kapeldreef 75, 3001 Leuven, Belgium \\ ${ }^{\mathrm{c}}$ Semiconductor Physics, Dept. of Physics, KU Leuven, Celestijnenlaan 200D, 3001 Leuven, \\ Belgium \\ ${ }^{\mathrm{d} S o l i d ~ S t a t e ~ P h y s i c s ~ a n d ~ M a g n e t i s m, ~ D e p t . ~ o f ~ P h y s i c s, ~ K U ~ L e u v e n, ~ C e l e s t i j n e n l a a n ~ 200 D, ~} 3001$ \\ Leuven, Belgium
}

\begin{abstract}
Because the germanium native oxide constitutes a poor dielectric, building metal oxide semiconductors (MOS) gate stacks on Ge requires passivation of the interface between the dielectric and the Ge channel. Different approaches to perform this passivation are available: $\mathrm{GeO}_{2}$ growth prior to high-k depositing, sulphur passivation, etc. The interface properties of these MOS stacks are important, because they determine the electrical properties of the whole structure. Dangling bonds introduce extra energy levels within the band gap, which results in a loss of efficiency in switching a MOS - field effect transistor on and off. Fixed charges near the interface enlarge the voltage needed for switching between on and off state as well. Hence, characterizing these interfaces is a key challenge in semiconductor fabrication. This can for example be achieved using Second Harmonic Generation (SHG) to probe the interface, because SHG is an inherent surface and interface sensitive technique. In this work, we present SHG as an promising surface and interface characterization tool for semiconductors for passivated germanium samples. Different SHG responses are shown for germanium samples with a sulphur passivated Ge or high-k dielectric on top of $\mathrm{Si}$. We show that the oxide layer as such is not probed by SHG and that different bonds over the Ge/oxide interface result in a difference SHG response.
\end{abstract}

Keywords: Interface, Metal oxide semiconductor, Germanium, Passivation methods, Characterization technique

\section{INTRODUCTION}

Since the dawn of semiconductor devices, there has been a driving force to shrink the dimensions of transistors. This enabled a drastic increase in the number of transistors that can be implemented in an integrated circuit (IC), which enhanced drastically the usefulness of a transistor. This trend is described as Moore's law: every 2 years, the number of transistors approximately doubles. ${ }^{1,2,3}$ In other words, the performance of an IC doubles every two years. This law is nowadays used as a guideline for technological challenges in semiconductor industry. In addition to Moore's law, an ITRS (international technology roadmap for semiconductors) roadmap ${ }^{4}$ is developed to specify the research topics in transistor optimization. This ensures that all participants who are working on the improvement of semiconductor devices, are striving for the same goals.

To increase the number of transistors on one IC, the dimensions of each individual transistor should decrease. Hence, one of the proposed research topics aims at reducing the channel length. The channel length is the distance between the source and the drain, as it is shown in Figure 1a. This is the area of the transistor which can be electrically screened to provide current through the device or to prevent a current from flowing through the device. The transistor performance heavily relies on the condition of the interfaces in the gate stack close to the channel. Therefore, it is of great importance 
to understand how the different manufacturing steps influence the interfaces in semiconductor devices and how these process steps influence the electrical passivation of a device. To keep up with the performance enhancement, alternative channel materials were investigated to replace $\mathrm{Si}$ in standard metal-oxide-semiconductor (MOS) devices. ${ }^{5}$ The use of high mobility material in the channel enhances the "on" current of a semiconductor device. For pMOS, Ge seems to be an ideal candidate. ${ }^{6}$ However, the implementation of Ge in semiconductor devices cannot be taken for granted. Passivation of Ge has been shown to be a serious issue. ${ }^{7}$ As opposed to $\mathrm{Si}$, Ge does not form a thermally stable native oxide $\left(\mathrm{GeO}_{2}\right)$. Moreover, the $\mathrm{Ge}-\mathrm{O}$ bonds (bond-dissociation energy of $157 \pm 5 \mathrm{kcal} / \mathrm{mol}$ ) are weaker than Si-O bonds (bond-dissociation energy of $185 \pm 7 \mathrm{kcal} / \mathrm{mol})^{8}$, resulting in a weaker $\mathrm{Ge}-\mathrm{O}$ network in the native oxide layer compared to $\mathrm{SiO}_{2}$. Most of all, hydrogen passivation to optimize the interface has little influence on the electrical or crystallographic properties of a Ge interface. ${ }^{9}$ Hence, additional processing steps have to be undertaken to deposit a dielectric or gate oxide layer.

Another research topic which is specified in the ITRS roadmap is the reduction of the gate oxide thickness or equivalent oxide thickness (EOT). The reduction in oxide thickness increases the oxide capacitance, which increases the "on" current of the device. Consequently, narrowing the oxide thickness has a positive influence on the device performance. However, upon decreasing the oxide thickness, leakage currents, due to a tunneling current, drastically increase. For a silicon oxide gate dielectric, tunneling current appears when the $\mathrm{SiO}_{2}$ layer is reduced to approximately $2 \mathrm{~nm}$. When implementing an oxide layer with higher dielectric constant than $\mathrm{SiO}_{2}$, the same capacitance can be retained for a thicker oxide layer, which also reduces the leakage currents. Both $\mathrm{HfO}_{2}$ and $\mathrm{Al}_{2} \mathrm{O}_{3}$ are thus being considered as replacement for $\mathrm{SiO}_{2} .{ }^{10}$ Another major research topic is sulfur passivation of Germanium. ${ }^{11}$ The implementation of both an alternative channel material and an alternative oxide layer brings additional challenges to semiconductor research as well as to characterization techniques. ${ }^{12}$ Any electrical defects at the interface screen the channel below and induce a delay in switching the semiconductor device on or off. Hence, improvement in understanding or production of the passivation layer is of great importance. More specifically, proper passivation, segregation, defects which induce energy levels within the band gap and reduce the efficiency of the semiconductor and fixed charges at the insulator/semiconductor interface are amongst the largest difficulties in semiconductor processing.

To overcome these difficulties, surface and interface specific characterization techniques are employed in semiconductor research to optimize transistors. A technique such as second-harmonic generation (SHG) is highly suited for investigation of interfaces in semiconductors. SHG is forbidden in centrosymmetric media within the dipole approximation. Centrosymmetry is broken at surfaces and interfaces in elemental semiconductors, resulting in a high interest for implementation of SHG in semiconductor research. In literature, it has been reported that SHG is a valid tool for studying interfaces in semiconductors. ${ }^{13}$ Most of the research has been done on Si. Rotational anisotropy SHG on Si provides insight in surface and interface properties, such as strain ${ }^{14,15}$, roughness ${ }^{16,17}$ and surface reconstruction. ${ }^{18}$ When including electric field induced second-harmonic generation (EFISHG), the electric field near the interface from externally applied voltage ${ }^{19}$ or doping $^{20}$ can be characterized.

In this publication, we combine the novel approaches in semiconductor research with the high sensitivity of secondharmonic generation. We have investigated MOS structures with different oxide thickness. A Si wafer was passivated with an $\mathrm{Al}_{2} \mathrm{O}_{3}$ oxide layer and those structures give all the same magnitude of isotropic SHG response, meaning that the oxide layer is not probed in an SHG measurement. Furthermore, we investigated a sulfur passivated Ge substrates with a $\mathrm{Al}_{2} \mathrm{O}_{3}$ gate oxide layer grown in different oxidation conditions, namely water and ozone. We show that SHG distinguishes a pure sulfur passivated Ge wafer from a sulfoxide passivated Ge wafer, which results from the different oxidizing agents during the ALD process.

\section{GENERAL CONCEPTS}

The operation of a transistor is key in understanding the goals which are specified in the ITRS roadmap. As can be seen from Figure 1a, a transistor consists of a source, drain and gate area, where the channel is located directly under the gate oxide. The sequence of the layers in a transistor is responsible for its name: Metal (gate) - Oxide (gate oxide) Semiconductor (substrate). In silicon or germanium-based transistors, the source and drain consist of negative or positive doped material. The body is oppositely doped compared to the source and drain. The oxide layer prevents the creation of 
a short circuit in the device. For use in transistor devices, the oxide layer should not contain any charges, which ensures a rapid screening of the channel and no power loss when turning a device "on" or "off".

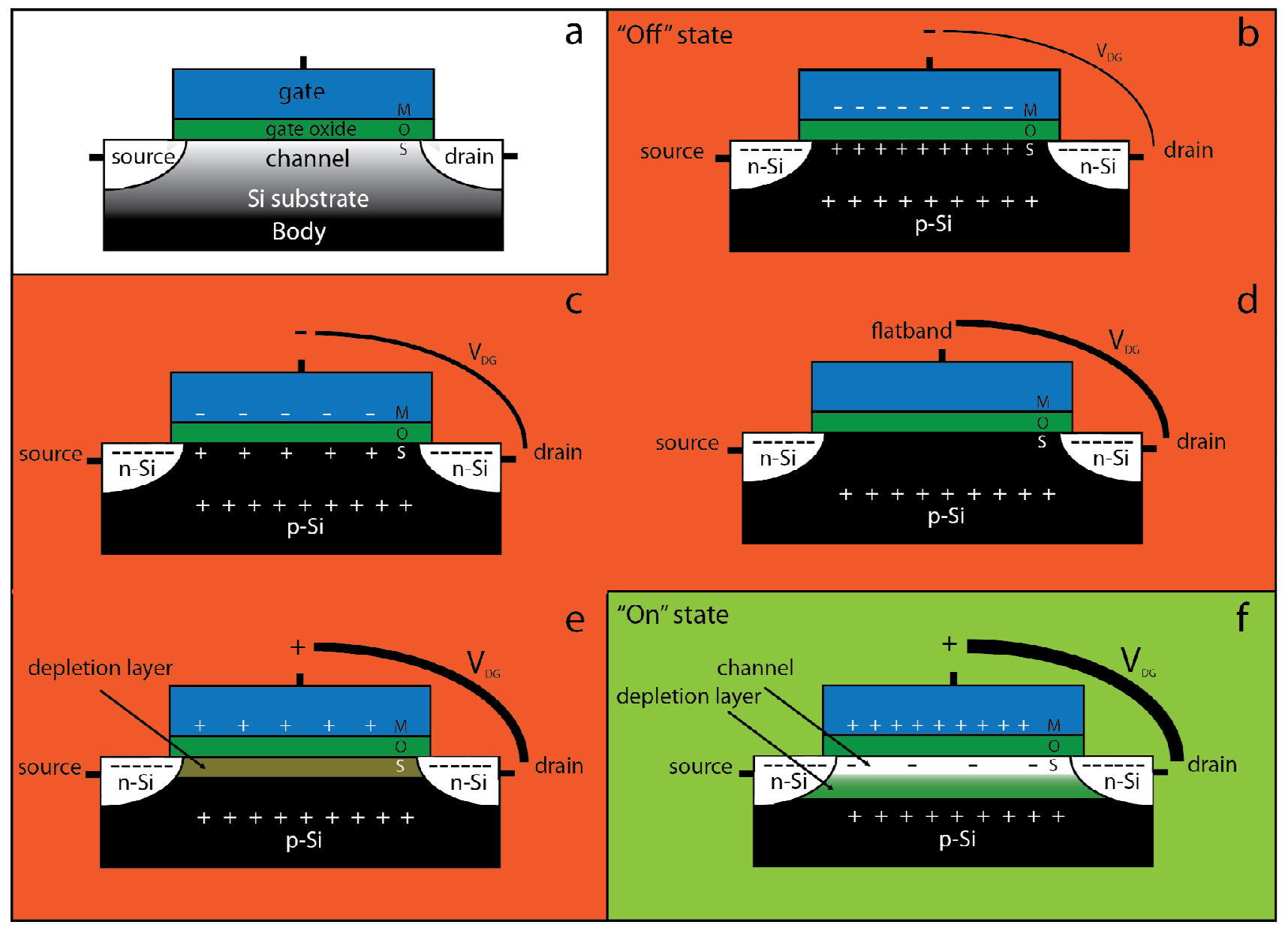

Figure 1: Representation of the working principle of a pMOS transistor. The different areas in a semiconductor device are shown in $a$. From $b$ to $f$, the state of the MOS device is shown for increasing voltage from the gate to the drain. From $\mathrm{b}$ to e, the device is in the "off" state, while the "on" state is depicted in $\mathrm{f}$.

By supplying a voltage from the gate to the drain, a conduction channel can be formed, depending on the sign and magnitude of the voltage. The doping level of a semiconductor device is always specified because it determines the operation of the device. The doping can be positive in the source and drain area (p-type transistor) or negative (n-type transistor). In the case of a n-doped channel (electrons flow through the channel), the source and drain are n-doped and the body is p-doped, see Figure 1b. When the device is turned "on", a current can flow from the source to the drain through the channel. When the device is turned "off", there is no conducting path between the source and the drain of the transistor. More specifically, when applying a negative voltage over the gate and drain, positive ions will be attracted to the gate/body interface, preventing a "negative" current from flowing from source to drain. The accumulation of the positive charges near the interface is called the accumulation layer (shown in Figure 1b), because the majority carriers of the body are accumulated there. However, when the voltage on the gate is increased, less holes (positive charges) are attracted to the interface between the body and the passivation layer, which reduces the thickness of the accumulation layer. (Shown in Figure 1c) At a certain voltage, which is called the flatband voltage, no net charges are present at the body/oxide interface. In this situation, the accumulation layer has completely disappeared, which is demonstrated in Figure 1d. When increasing the voltage even further, positive charges are inserted from the gate towards the oxide layer. Due to the electrostatic repulsion, the holes are pushed away from the oxide/body interface, which results in a depletion 
layer, where no more deficit of electrons is present. (Figure 1e) No negative charges are present due to the presence of majority carriers in the area, i.e. holes. If the voltage is increased again, majority charges are even further removed from the interface and a so-called inversion layer of channel is formed. In the channel, minority carriers are present and enable a current flow between the source and the drain. (Figure 1f) At this moment, the device is turned "on". Hence, changes in supply voltage, modulate the current through the transistor. As can be noticed, the interfaces where charges can accumulate are of greatest importance in a semiconductor device. The main goal is to understand and optimize these interfaces.

\section{METHODOLOGY}

The rotational anisotropy second-harmonic generation experiment was carried out using a $\mathrm{Ti}_{1}-\mathrm{Al}_{2} \mathrm{O}_{3}$ laser at the fixed fundamental wavelength of $800 \mathrm{~nm}$ with an average power output of $70 \mathrm{~mW}$. The pulse length and repetition rate are respectively $120 \mathrm{fs}$ and $82 \mathrm{MHz}$. The polarization of the incident beam on the sample is controlled by a polarizer and half-wave plate. This configuration allows us to adjust the polarization of the incident beam. In the experiment, the input polarization can be p-polarized or s-polarized, meaning that the electric field of the linearly polarized laser beam is parallel or perpendicular to the plane of incidence, respectively. The incident beam was cleared of the SHG originating in the laser cavity by a RG665 filter and then focused on the sample to a spot of approximately $40 \mu \mathrm{m}$. The angle of optical incidence is $45^{\circ}$ on the sample, which can be rotated around its surface normal. The generated photons of the half wavelength $(400 \mathrm{~nm})$ are then filtered trough two BG39 filters, which blocks the fundamental beam. An analyzer is placed after the sample, to vary the output second-harmonic beam. This output beam is detected with a photo multiplier tube and analyzed with a photon counter.

Within the electric dipole approximation, SHG is forbidden in centrosymmetric media, ${ }^{21}$ that is to say, it is only allowed in the case of broken symmetry. Symmetry in the material can be broken by externally applying electric ${ }^{22}$ or magnetic $^{23}$ dc fields, though care should be taken while estimating the dependence of the SHG signal on the latter. ${ }^{24}$ Moreover, the technique is also capable of imaging ferroelectric ${ }^{25}$ and ferromagnetic ${ }^{26}$ domains within the material. Additionally, chiral geometry $^{27}$ as well as the surface and interfaces in a multilayered system constitute symmetry breaking and all can give rise to dipolar SHG signal. In the latter case, the technique exhibits spectacular sensitivity, down to the atomic monolayer. ${ }^{28}$ For this reason, surface/interface specific properties, such as plasmon field enhancements ${ }^{29,30}$ or charge accumulation can be readily investigated by SHG. However, not all SHG signal is of dipolar origin and additional, higher multipoles should be taken into account. ${ }^{31}$ Hence, for intense incident electromagnetic fields, the polarization of the medium can be described by

$$
P(2 \omega)=P^{D}(2 \omega)+P^{Q}(2 \omega)=\chi^{D}: \mathbf{E}(\omega) \mathbf{E}(\omega)+\chi^{Q}: \mathbf{E}(\omega) \nabla \mathbf{E}(\omega),
$$

where $\omega$ the frequency of the light, $\mathbf{E}(\omega)$ and $\mathbf{E}(\omega)$ the electric fields vectors of the incident light and $\chi^{D}$ and $\chi^{Q}$ indicate the dipolar and the quadrupolar nonlinear susceptibilities, respectively. The dipolar nonlinear susceptibility $\chi^{D}$ is a third rank tensor with 27 components. However, the number of contributing components is reduced by symmetry considerations of the sample. ${ }^{32}$ Additionally, by changing the polarizer-analyzer configuration, different tensor components can be addressed. For a (001) oriented substrate, e.g. $\mathrm{Si}(001)$ and $\mathrm{Ge}(001)$ which are used in the investigated samples, the $\mathrm{S}_{\mathrm{in}}-\mathrm{P}_{\text {out }}$ polarizer-analyzer configuration, in $\chi^{D}$ a single tensor element is addressed, namely $\chi_{z y y}:$ the electric dipolar contribution is therefore a constant.

Despite the centrosymmetry, the bulk of the sample will contribute to the total signal as well through electric-quadrupole or magnetic dipole interactions. This nonlocal response, which corresponds to the last term in Equation 1, can be described by a quadrupolar polarization of the form: ${ }^{33}$

$$
{ }_{P}^{Q}(2 \omega)=(\delta-\beta-2 \gamma)(\boldsymbol{E} \cdot \nabla) E+\beta E(\nabla \cdot \boldsymbol{E})+\gamma \nabla(\boldsymbol{E} \cdot \boldsymbol{E})+\zeta \sum_{i} \hat{e}_{i} E_{i} \nabla_{i} E_{i}
$$


where $\beta, \delta, \gamma$ and $\zeta$ are phenomenological constants. We omitted the frequency dependence symbol $\omega$ of the fundamental fields for clarity reasons.

In a typical SHG experiment, the sample is rotated around its surface normal and the second-harmonic intensity is recorded for a certain input-output polarization. For the $\mathrm{S}_{\mathrm{in}}-\mathrm{P}_{\text {out }}$ polarizer-analyzer configuration, the azimuthal rotation can be phenomenologically described by: ${ }^{34,35}$

$$
I_{S_{\text {in }}-P_{\text {out }}}(2 \omega) \propto\left|I_{0}(2 \omega)\right|+2|B| \sin ^{2} 2(\phi+\theta)+|A| \sin ^{4} 2(\phi+\theta)
$$

where $I_{0}(2 \omega)$ is a combination of all isotropic terms squared from the dipolar and quadrupolar susceptibility, while A is the squared anisotropic contribution from the quadrupolar susceptibility. B is the product of the isotropic and anisotropic terms. In our results, the A term was measured in $S_{i n}-S_{\text {out }}$ and was found to be very small, consequently it was neglected in the fitting of the data. $\varphi$ is the azimuthal rotation of the sample and $\theta$ is a phase factor. We set $\varphi=0$ along the [110] direction of the crystal.

\section{RESULTS AND DISCUSSIONS}

Can the thickness of the oxide itself influence the SHG response? It is known that, upon growing an oxide layer, after a certain thickness of oxide layer is achieved, the morphology of the lower interface does not change. In this study, we investigated the SHG response of thick oxide layers. In Figure 2, the isotropic contribution of the total SHG signal is plotted as function of the oxide thickness of $\mathrm{Al}_{2} \mathrm{O}_{3}$ oxide layer on top of a $\mathrm{SiO}_{2}$ passivated $\mathrm{Si}$ wafers. On the silicon wafers, a chemical $\mathrm{Si}$ oxide of $1.1 \mathrm{~nm}$ was grown on top of the wafer. Thereafter, aluminium was deposited in an ALD reactor using the $\mathrm{Al}\left(\mathrm{CH}_{3}\right)_{3} / \mathrm{H}_{2} \mathrm{O}$ process, resulting in the final structure of $\mathrm{Al}_{2} \mathrm{O}_{3}$ on top of a $\mathrm{Si}$ wafers with a $\mathrm{SiO}{ }_{2}$ surface. Different thickness of $\mathrm{Al}_{2} \mathrm{O}_{3}$ was deposited, ranging from 10 to $30 \mathrm{~nm}$. From Figure 2, it can be seen that the isotropic contribution does not change when varying the oxide thickness. Hence, the oxide is not probed as such by SHG.

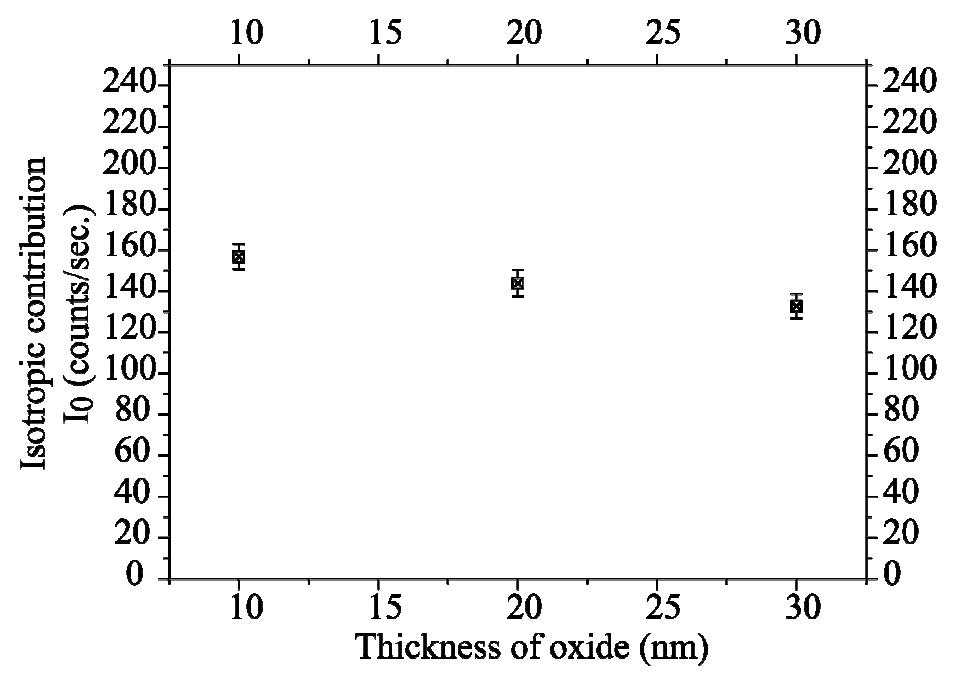

Figure 2: The isotropic contribution of an $\mathrm{Al}_{2} \mathrm{O}_{3}$ layer on top of partially oxidized $\mathrm{Si}$ as function of oxide thickness.

In Figure 3, the SHG response in the $\mathrm{S}_{\mathrm{in}}-\mathrm{P}_{\text {out }}$ polarizer-analyzer configuration of sulphur passivated Ge samples is shown. The samples at study consist of a p-type germanium wafer which was Ga doped with a resistivity of 0.04 to $0.4 \Omega \mathrm{m}$. These wafers were cleaned in an HF solution, removing most of the oxide on top of the Ge. Thereafter, the samples were annealed at $650{ }^{\circ} \mathrm{C}$ in hydrogen gas atmosphere for 10 minutes. Subsequently, the Ge substrate was exposed to $\mathrm{H}_{2} \mathrm{~S}$ at 20 
Torr, enabling the formation of 1 atomic layer of sulphur passivation layer. Finally, $\mathrm{Al}_{2} \mathrm{O}_{3}$ was deposited using the $\mathrm{ALD}$ growth mechanism of $\mathrm{Al}\left(\mathrm{CH}_{3}\right)_{3} / \mathrm{H}_{2} \mathrm{O}$ or $\mathrm{Al}\left(\mathrm{CH}_{3}\right)_{3} / \mathrm{O}_{3} .{ }^{10}$ The different behaviour of these two structures, with different oxide thickness, are investigated with SHG. The SHG response from bulk Si and bulk Ge is similar, due to the same symmetry consideration of the bulk materials, which enables comparison with the previous Si substrates.

It can be observed that the trend is similar for both materials. The SHG responses of both materials initially increases and thereafter the response decreases and stays approximately constant. However, the magnitude of $\mathrm{I}_{0}$, the isotropic contribution in the SHG signal is different for different oxidizing agents during the ALD process, namely ozone treatment or water treatment. Sioncke et $a l .{ }^{11}$ revealed that the structures grown with water or ozone as oxidizing agents in the ALD process differ in both electrical properties and morphology at and near the interface. We believe that the difference in the isotropic contribution of both sample series is arising from the difference in nature of the morphology of the interface. When performing the ALD process with water as oxidizing agent, the interface which is formed is a pure $\mathrm{S}$-passivated $\mathrm{Ge}$ interface from which the $\mathrm{Al}_{2} \mathrm{O}_{3}$ is starting to grow in a rather uniform manner. ${ }^{36}$ However, when using ozone as the oxidizing agent, both $\mathrm{Ge}-\mathrm{S}-\mathrm{Al}_{2} \mathrm{O}_{3}$ and $\mathrm{Ge}-\mathrm{O}-\mathrm{Al}_{2} \mathrm{O}_{3}$ bonds at the interface are present. Even the presence of Ge-O-S-O- $\mathrm{Al}_{2} \mathrm{O}_{3}$ or Ge-S-O- $\mathrm{Al}_{2} \mathrm{O}_{3}$ bonds cannot be excluded. This difference in material can be correlated with the difference in SHG response. A difference in material yields a different susceptibility tensor, resulting in a different isotropic behaviour. It can be seen that for larger numbers of ALD steps (or thicker oxide layers) the isotropic SHG response is approximately constant, which is consistent with the case of $\mathrm{Al}_{2} \mathrm{O}_{3}$ passivated Si. The precise physical mechanism behind this trend will be the subject of future research.

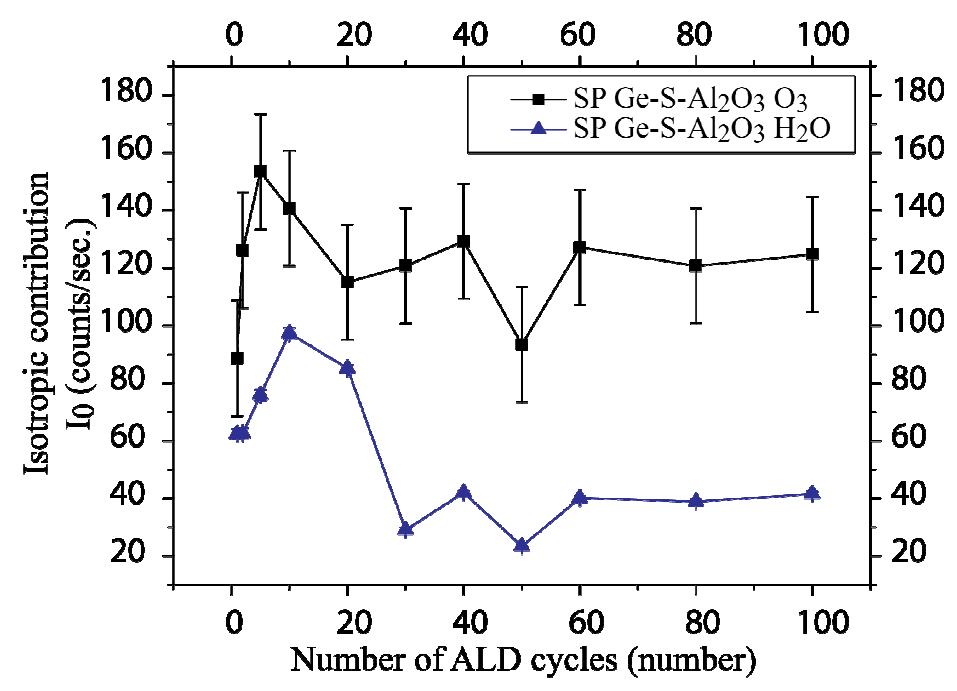

Figure 3: The isotropic contribution of $\mathrm{Ge}-\mathrm{S}-\mathrm{Al}_{2} \mathrm{O}_{3}$ structures grown with ozone (squared) or water (triangles) is shown in function of the number of ALD steps.

\section{CONCLUSION}

In this study, we provide evidence that the oxide layer of a MOS transistor is not probed in a rotational anisotropy SHG measurement. We report the same trend in isotropic SHG response from samples with different oxide thickness. Furthermore, it can be stated that the oxidizing agent determines the final structure of a MOS transistor. The difference in structure of the interface, arising from different oxidizing agents can be detected by SHG. The use of water as oxidant in the ALD process, results in a lower SHG response of the interface, compared to the sample which was prepared with ozone as the oxidizing agent. At this stage of out investigations, this can be attributed to the difference in bonds, which are present at the interface: the semiconductor is passivated with a pure sulphur interface when water is used as oxidizing agent, while Ge-S- $\mathrm{Al}_{2} \mathrm{O}_{3}, \mathrm{Ge}-\mathrm{O}-\mathrm{Al}_{2} \mathrm{O}_{3}$, Ge-S-O- $\mathrm{Al}_{2} \mathrm{O}_{3}$ and $\mathrm{Ge}-\mathrm{O}-\mathrm{S}-\mathrm{O}-\mathrm{Al}_{2} \mathrm{O}_{3}$ bonds appear when ozone is the oxidant. 


\section{ACKNOWLEDGEMENTS}

This work was supported by the Katholieke Universiteit Leuven (GOA). M.K.V. acknowledges the financial support from imec and the opportunity for collaboration. M.K.V. is very grateful for the support and scientific discussions with Ventsislav K. Valev. M.K.V. is thankful for the library services of Kevin Bogaerts.

\section{REFERENCES}

[1] Vincent, B., Damlencourt, J. F., Morand, Y., Pouydebasque, A., Le Royer, C., Clavelier, L., Dechoux, N., Rivallin, P., Nguyen, T. and Cristoloveanu, S., "The Ge condensation technique: A solution for planar SOI/GeOI co-integration for advanced CMOS technologies?," Mater. Sci. Semicond. Process. 11(5), 205-213 (2008).

[2] Moore, G., "Progress in digital integrated electronics," EDM 1.3, 11-13 (1975).

[3] Dennard, R.H., Gaensslen, F.H., Yu, H., Rideout, V.L., Bassous, E. and LeBlanc, A.R., "Design of ion-implanted MOSFET’s with very small physical dimensions," IEEE J. Solid-State Circuits 9(5), 256-268 (1974).

[4] http://www.itrs.net/reports.html

[5] Brunco, D.P., De Jaeger, B., Eneman, G., Mitard, J., Hellings, G., Sata, A., Terzieva, V., Souriau, L., Leys, F.E., Pourtois, G., Houssa, M., Winderickx, G., Vrancken, E., Sioncke, S., Opsomer, K., Nicholas, G., Caymax, M., Stesmans, A., Van Steenbergen, J., Mertens, P.W., Meuris, M. and Heyns, M.M., "Germanium MOSFET devices: Advances in materials understanding, process development, and electrical performance," Electrochem. Soc. 155(7), H552-H561 (2008).

[6] Shang, H., Okorn-Schmidt, H., Ott, J., Kozlowski, P., Steen, S., Jones, E.C., Wong, H.S. and Hanesch, W., "Electrical characterization of germanium p-channel MOSFETs," IEEE Electron Device Lett. 24(4), 242-245 (2003).

[7] Caymax, M., Eneman, G., Bellenger, F., Merckling, C., Delabie, A., Wang, G., Loo, R., Simoen, E., Mitard, J., De Jaeger, B., Hellings, G., De Meyer, K., Meuris, M. and Heyns, M., "Germanium for advanced CMOS anno 2009: a SWOT analysis," IEDM 19(3), 461-464 (2009).

[8] Dean, J. A., [CRC handbook of chemistry and physics: $85^{\text {nd }}$ edition] CRC press, New York, (1998).

[9] Afanas'ev, V.V., Fedorenko, Y.G. and Stesmans, A., "Interface traps and dangling-bond defects in (100)Ge/HfO2," Appl. Phys. Lett. 87(3), 032107 (2005).

[10] Chui, C., Ramanathan, S., Triplett, B.B., McIntyre, P. and Saraswat, K.C., "Germanium MOS capacitors incorporating ultrathin high-k gate dielectric," IEEE Electron Device Lett. 23(8), 473-475 (2002).

[11] Sioncke, S., Lin, H.C., Nyns, L., Brammertz, G., Delabie, A., Conard, T., Franquet, A., Rip, J., Struyf, H., De Gendt, S., Müller, M., Beckhoff, B. and Caymax, M., "S-passivation of the Ge gate stack: Tuning the gate stack properties by changing the atomic layer deposition oxidant precursor," J. Appl. Phys. 110(8), 084907 (2001).

[12] Vallett, D.P., "IC failure analysis: The importance of test and diagnostics," IEEE Des. Test. Comput. 14(3), 76-82 (1997).

[13] Downer, M.C., Mendoza, B.S. and Gavrilenko, V.I., "Optical second harmonic spectroscopy of semiconductor surfaces: Advances in microscopic understanding," Surf. Interface Anal. 31, 966-986 (2001).

[14] Meyer, C., Lüpke, G., Emmerichs, U., Wolter, F. And Kurz, H., "Electronic transistions at Si(111)/SiO2 and Si(111)/Si3N4 interfaces studied by optical second-harmonic spectroscopy,” Phys. Rev. Lett. 74(15), 3001-3004 (1995).

[15] Valev, V.K., Vanbel, M.K., Vincent, B., Moshchalkov, V.V., Caymax, M. and Verbiest, T., "Second harmonic generation indicates a better $\mathrm{Si} / \mathrm{Ge}$ interface quality for higher temperature and with $\mathrm{N} 2$ rather than with $\mathrm{H} 2$ as the carrier gas," IEEE Electron Device Lett. 32(1), 12-14 (2011).

[16] Dadap, J.I., Doris, B., Deng, Q., Downer, M.C., Lowell, J.K. and Diebold, A.C., " Randomly oriented Angstrom-scale microroughness at the $\mathrm{Si}(100) / \mathrm{SiO} 2$ interface probed by optical second harmonic generation," Appl. Phys. Lett. 64(16), 2139-2141 (1994).

[17] Cundiff, S.T., Knox, W.H., Baumann, F.H., Evans-Lutterodt, K.W., Tang, M.-T., Green, M.L. and van Driel, H.M., "Si/SiO2 interface roughness: Comparison between surface second harmonic generation and x-ray scattering," Appl. Phys. Lett. 70(11), 14141416 (1997).

[18] Heinz, T.F., Loy, M.M.T. and Thompson, W.A., "Study of Si(111) surfaces of optical second-harmonic generation: Reconstruction and surface phase transformation," Phys. Rev. Lett. 54(1), 63-66 (1985).

[19] Lüpke, G., "Characterization of semiconductor interfaces by second-harmonic generation," Surf. Sci. Rep. 35(3), 75-161 (1999).

[20] Lim, D., Downer, M.C. and Ekerdt, J.G., "Second-harmonic spectroscopy of bulk boron-doped Si(001)," Appl. Phys. Lett.77(2), 181-183 (2000).

[21] Verbiest, T., Clays, K. and Rodriguez, V., [Second-order nonlinear optical characterization techniques], CRC press Taylor \& Francis group, New York (2009).

[22] Lee, C. H., Chang, R.K. and Bloembergen, N., "Nonlinear electroreflectance in silicon and silver," Phys. Rev. Lett. 18(5), 167170 (1967). 
[23] Kirilyuk, A. and Rasing, Th., "Magnetization-induced-second-harmonic generation from surfaces and interfaces," J. Opt. Soc. Am. B 22(1), 148-167 (2005).

[24] Valev, V. K., Gruyters, M., Kirilyuk, A. And Rasing, Th., "Influence of quadratic contributions in magnetization-induced second harmonic generation studies of magnetization reversal," Phys. Stat. Sol. B 242, 3027-3031 (2005).

[25] Sheng, Y., Best, A., Butt, H.-J., Krolikowski, W., Arie, A. and Koynov, K., "Three-dimensional ferroelectric domain visualization by Cerenkov-type second harmonic generation," Opt. Express 18(16), 16539-16545 (2010).

[26] Pavlov, V. V., Ferré, J., Meyer, P., Tessier, G., Georgies, P., Brun, A., Beauvillain, P. and Mathet, V., "Linear and non-linear magneto-optical studies of Pt/Co/Pt thin films, “ J. Phys. Condens. Matter 13(44), 9867-9878 (2001).

[27] Valev, V. K., Zheng, X., Biris, C. G., Silhanek, A. V., Volskiy, V., De Clercq, B., Aktsipetrov, O. A., Ameloot, M., Panoiu, N. C., Vandenbosch, G. A. E. And Moshchalkov, V. V., "The origin of second harmonic generation hotspots in chiral optical metamaterials," Opt. Mater. Express 1, 36-45 (2011).

[28] Valev, V. K., Kirilyuk, A., Rasing, Th. Dela Longa, F., Kohlhepp, J. T. And Koopmans, B., “Oscillations of the net magnetic moment and magnetization reversal properties of the Mn/Co interface," Phys. Rev. B 75, 012401 (2007).

[29] Valev, V. K., Silhanek, A. V., De Clercq, B., Gillijns, W., Jevaram, Y., Zheng, X., Volskiy, V., Aktsipetrov, O. A., Vandenbosch, G. A. E., Ameloot, M., Moshchalkov, V. V. And Verbiest, T., "U-shaped switches for optical information processing at the nanoscale," Small 7, 2573-2576 (2011).

[30] Valev, V. K., Denkova, D., Zheng, X., Kuznetsov, A. I., Reinhardt, C., Chichkov, B. N., Tsutsumanova, G., Osley, E. J., Petkov, V., De Clercq, B., Silhanek, A. V., Jeyaram, Y., Volskiy, V., Warburton, P. A. Vandenbosch, G. A. E., Russev, S., Aktsipetrov, O. A., Ameloot, M., Moshchalkov, V. V. And Verbiest, T., "Plasmon-enhanced sub-wavelength laser ablation: plasmonic nanojets," Adv. Mater. 24, OP29-OP35 (2012).

[31] McGilp, J., "Optical characterization of semiconductor surfaces and interfaces,” Prog. Surf. Sci. 49(1), 1-106 (1995).

[32] Sipe, J.E., Moss, D. and van Driel, H.M., "Phenomenological theory of optical second- and third-harmonic generation from cubic centrosymmetric crystals," Phys. Rev. B 35(3), 1129-1141 (1987).

[33] Yang, S.A., Li, X., Bristow, A.D. and Sipe, J.E., "Second harmonic generation from tetragonal centrosymmetric crystals," Phys. Rev. B 80(16), 1-9 (2009).

[34] Vanbel, M.K., Valev, V.K., Vincent, B., Afanas'ev, V.V., Locquet, J.-P., Van Elshocht, S., Caymax, M. and Verbiest, T., "Second-harmonic generation reveals the oxidation steps in semiconductor prcessing," J. Appl. Phys., in press (2012).

[35] Falasconi, M, Andreani, L.C., Malvezzi, A.M., Patrini, M., Mulloni, V. And Pavesi, L., "Bulk and surface contributions to second-order susceptibility in crystalline and porous silicon by second-harmonic generation," Surf. Sci. 481(1), 105-112 (2001).

[36] Delabie, A., Sioncke, S., Rip, J., Van Elshocht, S., Caymax, M., Pourtois, G. and Pierloot, K., "Mechanisms for the trimethylaluminum reaction in aluminum oxide atomic layer deposition on sulphur passivated germanium," J. Phys. Chem. C. 112(35), 17523-17532 (2011). 